\title{
Alternativas terapêuticas na siringomielia
}

\author{
Flávio Freinkel Rodrigues*, Vinícius Teixeira Cimini**, Leonardo Mendes de Vuono**, \\ Elaine Marques Tinoco*** \\ Instituto de Neurologia Deolindo Couto (INDC) da Universidade Federal do Rio de Janeiro (UFRJ)
}

\section{RESUMO}

Os autores apresentam um estudo retrospectivo de dez pacientes com siringomielia tratados no Instituto de Neurologia Deolindo Couto e no Hospital Universitário Clementino Fraga Filho da UFRJ. São discutidos a fisiopatologia da doença e os vários tipos de técnicas cirúrgicas que são utilizadas no tratamento dessa doença.

Conclui-se que o tratamento cirúrgico da siringomielia ainda é uma questão aberta a novos estudos.

\section{PALAVRAS-CHAVE}

Siringomielia.

\section{ABSTRACT}

Therapeutic alternatives in syringomyelia

The authors present a retrospective study of a series of ten patients with syringomyelia.

The pathophysiology of the disease and the alternatives for surgical treatment are discussed.

\section{KEYWORDS}

Syringomyelia.

\section{Introdução}

O termo siringomielia originou-se da palavra grega syrinx, que significa tubo, e foi introduzida na literatura neurocirúrgica por Angers, em 1824³.

A primeira intervenção cirúrgica em pacientes com siringomielia foi atribuída a Abbe e Coley ${ }^{3}$, que realizaram uma mielotomia e retiraram líquido da cavidade da lesão medular, em $1892^{3}$.

Modernamente, Gardner ${ }^{6}$ dedicou-se ao estudo das lesões siringomiélicas e propôs, para o tratamento da siringomielia, a descompressão da fossa posterior com obstrução do óbex.

A siringomielia é definida estritamente como uma cavidade tubular na medula espinhal, que se estende por vários níveis. Esse termo não especifica a localização da cavidade em relação ao canal central da medula. Por essa razão, siringomielia é um termo inconclusivo, abrangendo diversas etiologias como hidromielia, cistos pós-traumáticos e cistos associados às alterações da junção craniovertebral.
O termo hidromielia, mais específico, foi criado por Simon e foi usado para descrever cavidades intramedulares originadas pela dilatação do canal central da medula, as quais contêm líquido idêntico ao líquido cefaloraquidiano $(\mathrm{LCR})^{13}$. Alguns autores sugerem o nome de hidrossiringomielia porque este inclui todas essas entidades.

A siringomielia é uma entidade clínica que pode ocorrer isoladamente, ou em associação com outras doenças neurológicas, como a malformação de Chiari, tumores, hemorragias ou secundariamente ao trauma.

As lesões cavitárias da medula espinhal são caracterizadas por sintomatologia complexa, e sofrem influência da etiologia, do tamanho e da localização da cavidade. Ainda é mal compreendido o papel da pressão intramedular, que depende do mecanismo hidrodinâmico do LCR.

Autores como Milhorat ${ }^{11}$, entre outros, acreditam que o acúmulo de líquido dentro da cavidade pode acentuar, ou desencadear, o déficit neurológico, por compressão do tecido medular.

\footnotetext{
* Professor-adjunto da Faculdade de Medicina da UFRJ, Doutor em Cirurgia, Coordenador-adjunto da Pós-graduação em Neurocirurgia. ** Médico residente em Neurocirurgia.

*** Acadêmica da Faculdade de Medicina da UFRJ.
} 
O objetivo deste trabalho é estudar as alternativas terapêuticas na siringomielia e os resultados obtidos com várias técnicas utilizadas. Fez-se uma revisão da literatura e estudou-se uma série de dez pacientes, cujos resultados serão aqui apresentados e discutidos. Não é nossa intenção propor novos tratamentos para a siringomielia, mas chamar a atenção de todos aqueles interessados no assunto de que as técnicas utilizadas devem ser revistas e que devemos discutir em conjunto sobre métodos terapêuticos mais eficientes.

\section{Apresentação da casuística}

Fez-se um estudo retrospectivo de dez pacientes com siringomielia (Quadro 1) e outras malformações associadas, atendidos no período de 1989 a 1999. Os pacientes foram selecionados entre aqueles que tiveram acompanhamento pós-operatório nos ambulatórios do Instituto de Neurologia Deolindo Couto (INDC) e do
Hospital Universitário Clementino Fraga Filho (HUCFF) da UFRJ. Os pacientes que foram escolhidos preenchiam os critérios de um protocolo elaborado previamente. Nesse protocolo foram observados o tempo da evolução da doença, outras malformações associadas, etiologia, tratamento cirúrgico e complicações.

Observou-se siringomielia na medula cervical em seis casos estudados $(60 \%)$ e, em quatro $(40 \%)$, na região toracolombar. Em seis casos $(60 \%)$ havia associação com malformações da junção craniovertebral (impressão basilar, Chiari tipo I e platibasia). A etiologia, em nove casos (90\%), foi idiopática e em apenas um (10\%), foi tumoral.

Realizou-se a derivação siringo-subaracnóidea em sete casos $(70 \%)$ e em três $(30 \%)$ foi realizada descompressão suboccipital e vertebral com duroplastia.

As complicações mais freqüentes foram fístula liquórica, observada em três casos $(30 \%)$, e infecção respiratória em dois casos $(20 \%)$.

Houve melhora da função motora em nove (90\%) dos dez pacientes tratados e, noutro restante, o quadro clínico manteve-se inalterado.

\begin{tabular}{|c|c|c|c|c|c|}
\hline \multicolumn{6}{|c|}{$\begin{array}{c}\text { Quadro I } \\
\text { Pacientes estudados no INDC e HUCFF }\end{array}$} \\
\hline Identificação & Diagnóstico & $\begin{array}{l}\text { Etiologia e tempo } \\
\text { de evolução }\end{array}$ & Operação & Complicações & Resultados \\
\hline $\begin{array}{l}\text { ICS, fem., } 50 \text { anos, } \\
\text { parda, RN }\end{array}$ & $\begin{array}{l}\text { Siringomielia } \\
\text { cervicotorácica }\end{array}$ & $\begin{array}{c}\text { Idiopática } \\
1 \text { ano e } 7 \text { meses }\end{array}$ & $\begin{array}{c}\text { Derivação } \\
\text { siringo-subaracnóidea }\end{array}$ & Fístula liquórica & $\begin{array}{l}\text { Melhora parcial } \\
\text { da motricidade }\end{array}$ \\
\hline $\begin{array}{l}\text { JML, masc., } 49 \text { anos, } \\
\text { branco, carpinteiro }\end{array}$ & $\begin{array}{l}\text { Siringomielia cervical } \\
\text { + invaginação basilar } \\
\text { + neurinoma }\end{array}$ & $\begin{array}{l}\text { Idiopática } \\
12 \text { anos }\end{array}$ & $\begin{array}{c}\text { Derivação } \\
\text { siringo-subaracnóidea }\end{array}$ & & $\begin{array}{l}\text { Melhora parcial } \\
\text { da motricidade }\end{array}$ \\
\hline $\begin{array}{l}\text { JCS, masc., } 40 \text { anos, } \\
\text { branco, arrumador, PE }\end{array}$ & $\begin{array}{l}\text { Siringomielia }+ \\
\text { Chiari tipo I }+ \\
\text { cifoescoliose }\end{array}$ & $\begin{array}{l}\text { Idiopática } \\
10 \text { anos }\end{array}$ & $\begin{array}{c}\text { Derivação } \\
\text { siringo-subaracnóidea } \\
\text { craniectomia suboccipital }\end{array}$ & & $\begin{array}{l}\text { Melhora parcial } \\
\text { da motricidade }\end{array}$ \\
\hline $\begin{array}{l}\text { ENE, fem., } 53 \text { anos, } \\
\text { branca, do lar, } \\
\text { Portugal }\end{array}$ & $\begin{array}{l}\text { Siringomielia cervical } \\
\quad+\text { siringobulbia }\end{array}$ & $\begin{array}{l}\text { Idiopática } \\
13 \text { anos }\end{array}$ & $\begin{array}{c}\text { Derivação } \\
\text { siringo-subaracnóidea }\end{array}$ & & $\begin{array}{l}\text { Melhora parcial } \\
\text { da motricidade }\end{array}$ \\
\hline $\begin{array}{l}\text { PCOS, masc., } 38 \text { anos, } \\
\text { negro, funcionário } \\
\text { público, RJ }\end{array}$ & $\begin{array}{l}\text { Siringomielia }+ \\
\text { astrocitoma T5-T9 }\end{array}$ & $\begin{array}{c}\text { Tumoral } \\
4 \text { anos }\end{array}$ & $\begin{array}{c}\text { Derivação } \\
\text { siringo-subaracnóidea }\end{array}$ & & $\begin{array}{l}\text { Melhora parcial } \\
\text { da motricidade }\end{array}$ \\
\hline $\begin{array}{l}\text { CJS, masc., } 30 \text { anos, } \\
\text { negro, servente, PB }\end{array}$ & $\begin{array}{l}\text { Siringomielia }+ \\
\text { Chiari tipo I }+ \\
\text { platibasia }\end{array}$ & $\begin{array}{l}\text { Idiopática } \\
5 \text { anos }\end{array}$ & $\begin{array}{l}\text { Craniectomia } \\
\text { suboccipital }\end{array}$ & Fístula liquórica & $\begin{array}{l}\text { Melhora parcial da } \\
\text { motricidade e } \\
\text { síndrome cerebelar }\end{array}$ \\
\hline $\begin{array}{c}\text { CC, fem., } 20 \text { anos, } \\
\text { branca, estudante, RJ }\end{array}$ & $\begin{array}{c}\text { Siringomielia }+ \\
\text { Chiari tipo I }\end{array}$ & $\begin{array}{l}\text { Idiopática } \\
3 \text { anos }\end{array}$ & $\begin{array}{c}\text { Derivação } \\
\text { siringo-subaracnóidea }\end{array}$ & Fístula liquórica & $\begin{array}{l}\text { Melhora parcial } \\
\text { da motricidade }\end{array}$ \\
\hline $\begin{array}{l}\text { JRCB, masc., } 36 \text { anos, } \\
\text { negro, servente, MA }\end{array}$ & $\begin{array}{l}\text { Siringomielia cervico- } \\
\text { torácica }+ \text { Chiari tipo I }\end{array}$ & $\begin{array}{l}\text { Idiopática } \\
10 \text { anos }\end{array}$ & $\begin{array}{l}\text { Craniectomia } \\
\text { suboccipital }\end{array}$ & Pneumonia & $\begin{array}{l}\text { Melhora parcial } \\
\text { da motricidade }\end{array}$ \\
\hline $\begin{array}{l}\text { MFA, fem., } 56 \text { anos, } \\
\text { branca, do lar, RJ }\end{array}$ & Siringomielia T3-L3 & $\begin{array}{l}\text { Idiopática } \\
4 \text { anos }\end{array}$ & $\begin{array}{c}\text { Derivação } \\
\text { siringo-subaracnóidea }\end{array}$ & & $\begin{array}{l}\text { Quadro clínico } \\
\text { inalterado }\end{array}$ \\
\hline $\begin{array}{c}\text { JLBV, masc., } 27 \text { anos, } \\
\text { motorista, RJ }\end{array}$ & $\begin{array}{l}\text { Siringomielia C4-C6 } \\
\quad+\text { Chiari tipo I }\end{array}$ & Idiopática & $\begin{array}{l}\text { Craniectomia } \\
\text { suboccipital }\end{array}$ & Pneumonia & $\begin{array}{l}\text { Melhora parcial } \\
\text { da motricidade }\end{array}$ \\
\hline
\end{tabular}




\section{Discussão}

A fisiopatologia da siringomielia está baseada no mecanismo de distensão da medula por coleção de LCR, que se comunica livremente com o canal central da medula e com o IV ventrículo.

A siringomielia está associada, em três quartos dos casos, à malformação de Chiari, ou a outras anomalias que ocluem o forame de Magendie. Baseados nesses achados, Gardner ${ }^{6}$ desenvolveu a teoria hidrodinâmica para explicar a evolução dessa afecção. De acordo com esse autor, a obstrução parcial ou completa do forame de Magendie é causada pela distensão do tubo neural. Essa distensão ocasionará dilatação cística da medula, resultando em uma dilatação lenta e progressiva do canal central. Formase, então, um divertículo que se origina do canal central da medula e se comunica com o espaço liquórico através do IV ventrículo.

Barnett ${ }^{2}$ concorda que a cavidade siringomiélica, ou hidromiélica, é a causa mais provável da síndrome central da medula na sua forma espontânea. Esse autor chama esta condição de siringomielia comunicante.

A teoria hidrodinâmica é ainda aceita, embora não tão bem compreendida, pelo fato de que a cavidade siringomiélica aumenta no diâmetro transverso da medula. A indicação da descompressão cirúrgica baseia-se nesse mecanismo fisiopatológico.

Milhorat e cols. ${ }^{11}$ encontraram apenas evidências indiretas de compressão medular e afirmaram que a fisiopatologia do aumento da cavidade siringomiélica não foi até aquele momento esclarecida.

Durante o ato cirúrgico, não são realizadas, de rotina, medidas da pressão intracavitária. As medidas do fluxo sangüíneo local da medula servem apenas para auxiliar no acompanhamento, dando uma noção do aumento da cavidade, carecendo de dados mais objetivos e de futuros estudos.

$\mathrm{O}$ tratamento da siringomielia está fundamentado em várias teorias que até o momento não tiveram comprovação. Algumas técnicas cirúrgicas que são realizadas não demonstram qualquer eficácia e, no decorrer de algum tempo após a intervenção, a doença agrava-se.

Entre as principais técnicas cirúrgicas encontramse a descompressão da fossa posterior com ou sem o fechamento do óbex ${ }^{4}$, a secção do filum terminale 5 , mielotomia e derivações do líquido da cavidade siringomiélica (lomboperitonial, siringopleural, siringosubaracnóidea, siringoperitonial ).

Lund-Johansen e cols. ${ }^{10}$ estudaram uma série de 18 pacientes com siringomielia e concluíram que a drenagem da cavidade deve ser feita com dreno reto e não com dreno em forma de "T", e que não é necessária a colocação de válvula no sistema de drenagem, principalmente nas cavidades de baixa pressão. Nessa série foi realizada a derivação siringopleural. Porém, vários autores como Barbaro $^{1}$, Lesoin ${ }^{8}$ e Phillips ${ }^{13}$ recomendam a inclusão de válvula no sistema de derivação.

Existe também discussão entre a técnica de derivação versus a descompressão da fossa posterior. Sgouros e Willians ${ }^{15}$ observaram que os pacientes com derivação siringopleural, na sua grande maioria, necessitam de uma segunda operação. No entanto, há concordância de que na falha da derivação, estaria indicada a descompressão da fossa posterior, quando houver malformação de Chiari associada ${ }^{6,10,12}$.

Iskandar e cols. ${ }^{7}$ estudaram 27 casos de hidrossiringomielia terminal, chamando a atenção para a associação desse tipo de lesão com o disrafismo espinhal oculto ( $69 \%$ dos casos). Os autores questionam as teorias que tentam explicar a fisiopatologia da hidrossiringomielia e afirmam que a apresentação clínica dessas lesões na medula terminal tem suas particularidades. O tratamento cirúrgico, segundo esses autores, deve ser proposto apenas para lesões maiores que $2 \mathrm{~cm}$ de diâmetro.

Lorenzo e cols. ${ }^{9}$ apresentaram uma série de 20 pacientes com siringomielia associada à malformação de Chiari tipo I tratados com descompressão craniovertebral. Esses autores denominam essa técnica de conservadora e a classificaram como menos invasiva, quando comparada as outras técnicas, porém com resultados semelhantes. Nessa série, os resultados obtidos foram: 8 pacientes melhoraram, 11 pacientes permaneceram estáveis e 1 paciente apresentou piora do quadro neurológico, apesar do colabamento da cavidade siringomiélica.

Milhorat e cols. ${ }^{11}$ propuseram um estudo com medidas da pressão da cavidade siringomiélica em pacientes com deterioração clínica progressiva e correlacionaram esses dados com os déficits neurológicos e com os achados fisiológicos intra-operatórios. Para esses autores, esse estudo foi o primeiro a medir a pressão da cavidade siringomiélica em medula humana. Eles encontraram cavidades distendidas associadas a vários níveis de aumento da pressão intramedular que induziriam a vários tipos de disfunções neurológicas pela compressão dos tratos longos, dos neurônios e da microcirculação. Esse quadro neurológico é potencialmente reversível pela descompressão cirúrgica da cavidade siringomiélica. As medidas da pressão intramedular foram obtidas por meio de shunt colocado dentro da cavidade siringomiélica, ligado a um manômetro. A pressão média foi medida no período entre as incursões respiratórias, utilizando a superfície dorsal da medula espinhal como ponto de referência. O estudo sugere que a pressão do líquido da cavidade siringomiélica varia de $0,5 \mathrm{~cm}$ de $\mathrm{H}_{2} \mathrm{O}$ a $22 \mathrm{~cm}$ de $\mathrm{H}_{2} \mathrm{O}$ (média de $7,7 \mathrm{~cm}$ de $\mathrm{H}_{2} \mathrm{O}$ ), quando medidas sob condições atmosféricas. 
Todos esses achados concluíram que o aumento da cavidade intramedular é resultante de uma força intramedular de suficiente magnitude que vence a resistência do tecido medular e da pressão do LCR no espaço subaracnóideo.

Wiedemayer e cols. ${ }^{17}$ relataram a experiência obtida em 22 pacientes com siringomielia tratados cirurgicamente, com diferentes tipos de derivações do líquido da cavidade. Em 7 casos foram realizadas derivações siringo-subaracnóidea e, em 8, a siringopleural. As outras cavidades foram drenadas para o espaço peritonial. Ocorreu melhora em 5 casos, 12 casos permaneceram inalterados, piora clínica em 1 caso e morte em 1 caso. Esse estudo levou os autores a concluírem que o tratamento cirúrgico deve preceder déficits neurológicos irreversíveis.

Nos 10 casos estudados nessa série, obteve-se melhora das parestesias, e, principalmente, da motricidade em $90 \%$ desses casos. No entanto, não foram observadas diferenças no resultado entre as duas técnicas empregadas: derivação siringo-subaracnóidea e descompressão craniovertebral com duroplastia. Devese salientar, no entanto, que a melhora foi transitória em cerca de $90 \%$ desses; ulteriormente, os déficits neurológicos retornaram com a mesma intensidade. Não tivemos condição de avaliar com precisão em quantos pacientes, e por quanto tempo, manteve-se a remissão do quadro clínico após o tratamento cirúrgico.

Entre os autores ${ }^{6,11,12}$ há concordância de que a fisiopatologia da siringomielia ainda não está suficientemente esclarecida para orientar a terapêutica.

\section{Conclusão}

O tratamento cirúrgico deve ser revisto, na tentativa de encontrar alternativas que possam melhorar o prognóstico desses pacientes.

Pode-se afirmar, pela nossa experiência e revisão da literatura, que o tratamento cirúrgico da siringomielia é ainda uma questão aberta a novos estudos e discussões.

\section{Referências}

1. BARBARO NM, WILSON CB, GUTIN PH, EDWARDS MSB: Surgical treatment of syringomyelia: favorable results with syringoperitoneal shunting. J Neurosurg 61:531-8, 1984

2. BARNETT HJM, FOSTER JB, HEDSON P: Syringomyelia. London, WB Saunders Company, 1973, pp 558-73.
3. BATZDORF U: Syringomyelia, Chiari malformation, and hydromyelia. In Youmans JR (ed): Neurological surgery. 4 ed. London, WB Saunders Company, 1996, pp 1090-109.

4. DYSTE GN, MENEZES AH, VANGILDER JC: Symptomatic Chiari malformation: an analysis of presentation, manegement, and long term outcome. J Neurosurg 71:159-68, 1989.

5. GARDNER WJ, BELL HS, POOLOS PN, DOHN DF, STEINBERG M: Terminal ventriculostomy for syringomyelia. J Neurosurg 46:609-17, 1977.

6. GARDNER WJ: Hidrodynamic mechanism of syringomyelia: its relationship to myelocele. J Neurol Neurosurg Psychiatry 28:247-59, 1965.

7. ISKANDAR BJ, OAKES WJ, MCLAUGHLIN C, OSUMI AK, TIEN RD: Terminal syringohidromyelia and occult spinal dysrafism. J Neurosurg 81:513-9, 1994.

8. LESOIN F, PETIT H, THOMAS CE, VIAUD C, BALERIAUX D, JOMIN M: Use of syringoperitoneal shunt in the treatment of syringomyelia. Surg Neurol 25:131-6, 1986.

9. LORENZO N, PALMA L, PALAITINSKY E, FORTUNA $A$ : Conservative cranio-cervical descompression in the treatment of syringomyelia-Chiari I complex: a prospective study of 20 adults cases. Spine 20:247983, 1995.

10. LUND-JOHANSEN M, WESTER K: Syringomyelia treated with a nonvalved syringoperitoneal shunt: a follow-up study. Neurosurgery 41:858-63, 1997.

11. MILLHORAT TH, CAPOCELLI AL, KOTZEN RM, BOLOGNESE P, HEGER IM, COTTRELL JE: Intramedullary pressure in syringomyelia: clinical and pathophysiological correlates of syrinx distension. Neurosurgery 41:1102-8, 1997.

12. OLDFIELD EH, MURASZKO K, SHAWKER TH, PATRONAS NJ: Pathophysiology of syringomyelia associated with Chiari I malformation of cerebelar tonsils: implications for diagnosis and treatment. J Neurosurg 80:3-15, 1994.

13. PHILLIPS TW, KINDT GW: Syringoperitoneal shunt for syringomyelia: a preliminary report. Surg Neurol 16:462-6, 1981.

14. RAUZZINO M, OAKES J: Chiari II malformation and syringomyelia. Neurosurg Clin N Am 6:293-309, 1995.

15. SGOUROS S, WILLIANS B: A critical appraisal of drainage in syringomyelia. J Neurosurg 82:1-10, 1995.

16. WESTER K, PEDERSEN PH, KRAKENES J: Spinal cord damage caused by rotation of a T-drain in a patient with syringoperitoneal shunt. Surg Neurol 31:224-7, 1989.

17. WIEDEMAYER H, NAU HE, RAUHUT F, GROTE W, GERHARD L: Operative treatment and prognosis of syringomyelia. Neurosurgery 17:37-41, 1994.

18. WILLIANS B: Syringomyelia and syringobulbia. Neurosurgery 5:229-51, 1995.

Original recebido em setembro de 1999

Aceito para publicação em março de 2000

\section{Endereço para correspondência:}

Flavio F. Rodrigues

Rua Buarque de Macedo, 14/606

CEP 22220-030 - Rio de Janeiro, RJ 